\title{
Methodology of testing and optimal setting of an asynchronous traction drive for electric vehicles
}

\author{
Vasil Dimitrov ${ }^{1, *}$ \\ ${ }^{1}$ Todor Kableshkov University of Transport - Sofia, Faculty of Telecommunications and Electrical Equipment in Transport, \\ Department of Power Engineering and Electrical Equipment in Transport, 1574 Sofia, 158 Geo Milev St., Bulgaria
}

\begin{abstract}
This paper presents a methodology of tests of traction asynchronous drives applied in electric vehicles. It is similar to Urban Driving Cycle ECE-15 (UDC). The objective of the research is to verify the developed methodology through tests performed on a laboratory simulator, which consists of energy-saving asynchronous motor controlled by a frequency inverter (converter). Vector Control on the drive is used for smooth speed regulation over a wide range at high values of efficiency and power factor. Contemporary measuring and control devices and special software products are used for capturing the dynamic characteristics when starting, stopping and changing the speed set point of the drive. Some of tests are presented and an experimental verification of the developed methodology is made. An optimal setting of the drive parameters can be made to minimize the electric energy consumption.
\end{abstract}

\section{Introduction}

Contemporary electric vehicles are designed on the basis of power drives controlled by highly efficient devices and microprocessor control and safety systems [1, 2]. Asynchronous motors with improved efficiency and performance are introduced into many modern trams, trolleybuses, underground trains and locomotives, as well as in hybrid and fully electric cars. These motors possess extremely long life and high reliability, offer considerable energy savings and protect the environment, their weightoptimized design has a positive effect on the stability of the equipment unit. Motors with aluminium housing have particular low weight and they are preferred for applications in vehicles. There are also motors with increased output and compact construction which could be used to advantage in confined spaces. They are also optimized for efficiency and designed for inverter-fed operation with a pulse width modulation (PWM). They feature an insulation system consisting of high-quality enamel wires and insulating sheeting in conjunction with solvent-free resin impregnation. A smooth speed control from 0 to several times over the rated one is limited only by the limits for the roller bearings, critical rotor speed and rigidity of the rotating parts.

In this paper, a methodology of testing traction asynchronous drives applied in electric vehicles is developed. It is similar to Urban Driving Cycle ECE-15 (UDC) used to assess the emission levels and fuel economy consumption of car engines, as well as for the measurement of electric energy consumption and electric range in hybrid and fully electric vehicles [3-6]. A laboratory system has been designed and built and contemporary measuring and devices are used for monitoring and control. The objective of the research is to verify the developed methodology through tests performed on the simulator using special software products to capture the dynamic characteristics when starting, stopping and changing the speed set point of the drive.

\section{Possibilities of speed and torque control on the traction drive}

In electric vehicles, load torque varies significantly and speed should be adjusted smoothly from zero to the maximum value. In this case, the primary law of frequency controlled asynchronous drives (established by M. P. Kostenko) must be used [1, 2]:

$$
\frac{U_{1}}{U_{1 N}}=\frac{f_{1}}{f_{1 N}} \sqrt{\frac{M}{M_{N}}},
$$

where $U_{1}$ and $U_{1 N}$ are the adjustable and nominal voltage; $f_{1}$ and $f_{1 N}-$ adjustable and nominal frequency; $M$ and $M_{N}$ - load torque and rated motor torque.

This method is known as "Vector Control" (Fieldorientated closed-loop control). It ensures reliable and flexible motor operation and provides a stable speed irrespective of any disturbances (like the supply pressure fluctuations, climatic conditions, load mass, road profile, track condition etc.). The Vector Control (VC) has also many other advantages: stable parameters when changing loads and setting point values, the motor and braking torque are controlled independently of the speed, accelerating and braking are possible with a maximum adjustable torque, high values of the power factor and the efficiency can be achieved. Therefore, the VC must be used in all applications where the load varies widely, as in

\footnotetext{
* Corresponding author: vdimitroff@abv.bg
} 
electric vehicles. The use of maximum adhesion, if necessary (e.g. starting or climbing a slope), and the realization of skid resistance is also possible.

\section{Methodology of testing}

\subsection{Driving cycles}

A driving cycle is a series of data points representing the speed of a vehicle versus time. Different driving cycles are developed to assess the performance of vehicles in various ways, as for example fuel consumption, polluting emissions, performance of internal combustion engines, transmissions, electric drive systems, batteries, fuel cell systems, and similar components [3-6]. The Urban Driving Cycle ECE-15 (or just UDC) has been designed to represent typical driving conditions in busy European cities (defined by ECE R83, R84 and R101). It is characterized by low engine load and a maximum speed of $50 \mathrm{~km} / \mathrm{h}$ (Fig. 1).

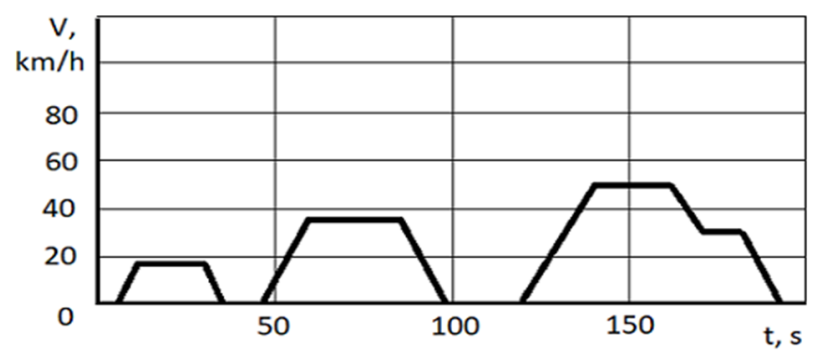

Fig. 1. Scheme of the Urban Driving Cycle (UDC).

\subsection{Laboratory simulator}

The laboratory simulator is built on contemporary devices, based on the newest technologies (Fig. 2) [7]. The main elements involved in the configuration of the simulator are developed by Siemens: three phase energysaving asynchronous motor (AM - 2,2 kW) with lightweight construction; Sinamics G120 frequency converter (includes three phase IGBT inverter and Microprocessor Control Unit - CU); Simatic S7-200 Programmable Logic Controller (PLC); TP-177micro Touch Panel. An encoder is mounted on the motor shaft and is used for closed loop motion control on the drive. A synchronous generator $(\mathrm{SG}-30 \mathrm{~V}, 60 \mathrm{~A})$ with a bridge rectifier and resistors simulates the load of the motor. It can be changed smoothly using the Flux current Regulator or resistors. A WINDOWS-based computer system is used for configuring and data storage. The necessary commissioning software STARTER is installed on this computer and is used for setting-up the Sinamics G120. A subprogram TRACE is developed for real-time tracing of the inverter parameters. It can trace up to 30 parameters simultaneously, and then export the obtained data into an Excel sheet and create graphs. The intelligent energy meter EMPS is connected to the drive and allows for monitoring the operation and analyzing the current consumption and power flows.

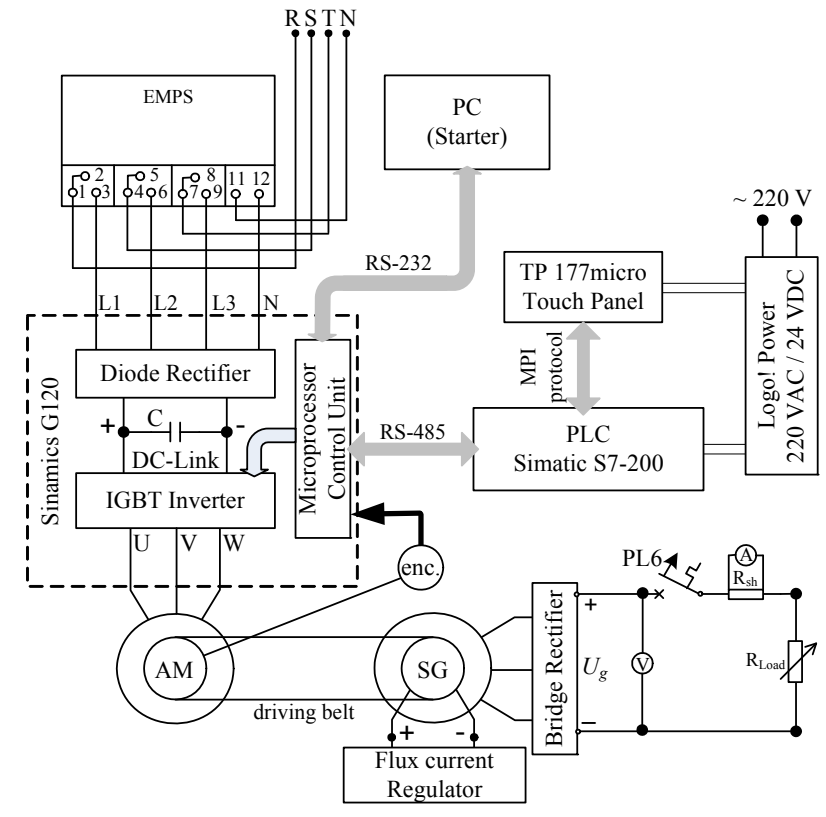

Fig. 2. Scheme of the laboratory simulator.

\subsection{Experimental research}

The software STARTER and the subprogram TRACE have to be run. VC is used and the load is constant during the tests (equal to $75 \%$ of the rated motor torque). The developed methodology consists of the following tests:

- The motor slowly accelerates to $450 \mathrm{rpm}$ (equivalent to $30 \%$ of the nominal speed $-15 \mathrm{~km} / \mathrm{h}$ ), runs at constant speed, and then slowly stops.

- After a short break the motor slowly accelerates to $960 \mathrm{rpm}$ (equivalent to $64 \%$ of the nominal speed $32 \mathrm{~km} / \mathrm{h}$ ), runs at constant speed, and then slowly brakes to a full stop.

- After a new short break the motor slowly accelerates to $1500 \mathrm{rpm}$ (equivalent to the nominal speed of $50 \mathrm{~km} / \mathrm{h}$ ), runs at constant speed, and then slowly stops (according to SAE J227a driving schedule the last element of the UDC is not necessary to be included when electric vehicles are tested - it could be replaced by a 'coast' phase, during which the tractive effort is set to zero but the speed is not specified, and the energy consumption is negligible [3]).

The obtained graphs are shown in Fig. 3.

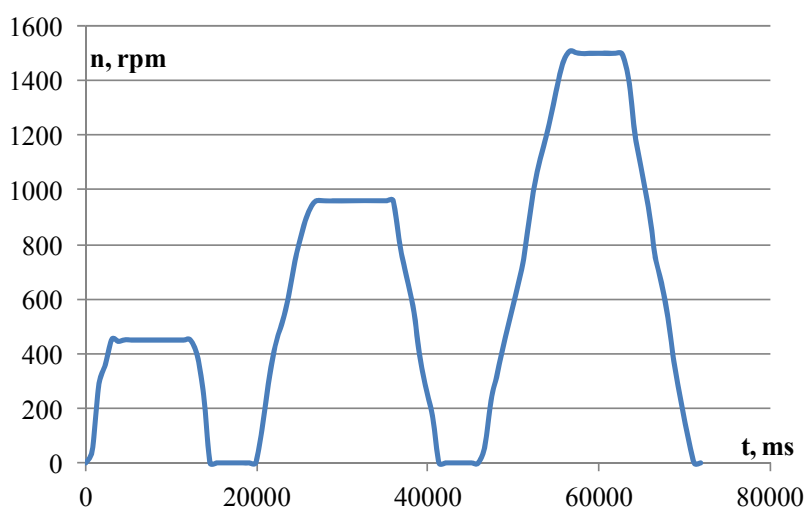

Fig. 3. Graph for the developed methodology. 


\subsubsection{Test results}

The speed set points, as well as the "Start" and "Stop" commands, are put by Touch panel and PLC [7]. Except for the actual rotor speed (shown in Fig. 3), the frequency, torque, power, voltage, power factor and current (as well as its components) have been traced by TRACE subprogram and STARTER commissioning software. Some of test results are shown in Figures 4, 5, and 6. The energy consumption has been measured by EMPS.

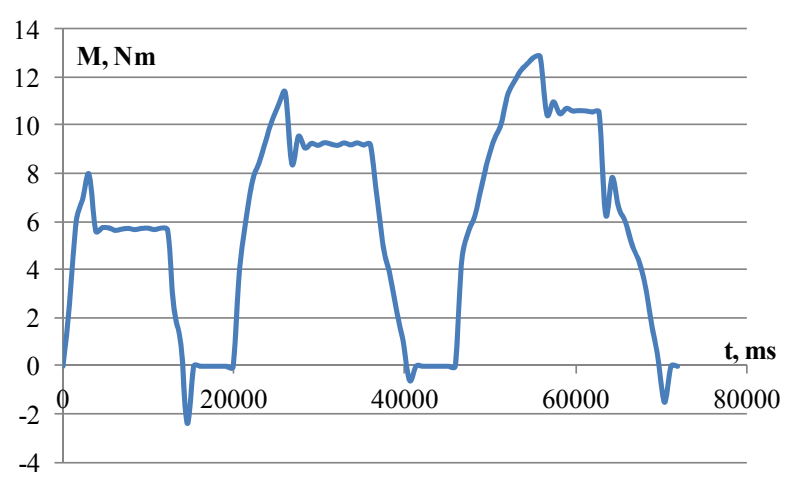

Fig. 4. The motor torque during the tests.

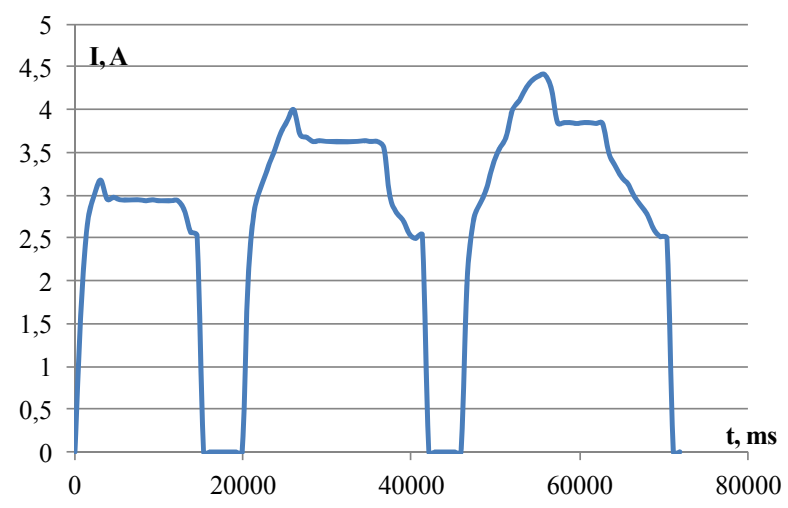

Fig. 5. The motor current during the tests.

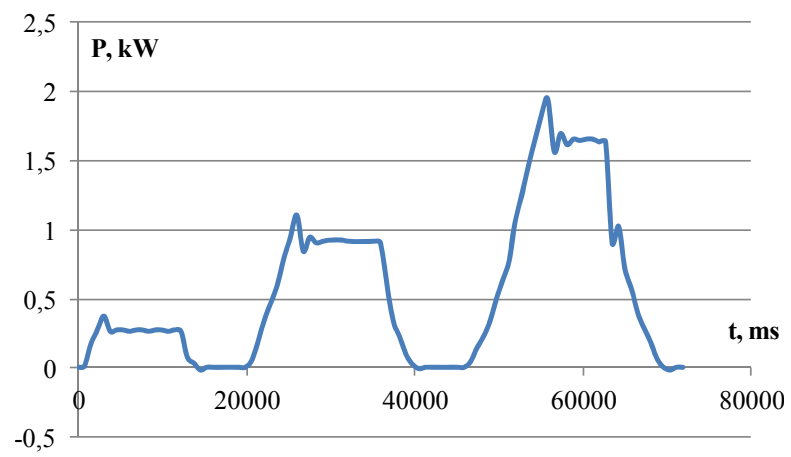

Fig. 6. The active power during the tests.

\subsubsection{Study of the impact of the acceleration time on the drive performance}

As opposed to the UDC, the acceleration and deceleration time can be changed and their impact on the drive performance and energy consumption can be examined. The ramp-function is used to limit the acceleration when the speed set point $n_{s p}$ (proportional to $f_{s p}$ ) changes according to a step function. This helps to reduce the stress on the mechanical system of the drive. An acceleration ramp can be set using parameter $T_{\text {ramp-up }}$ of Sinamics G120. This is the time it takes the motor to accelerate from standstill up to maximum motor frequency $f_{\max }$ when no rounding is used. This function allows also for a controlled transition when the set point is changed. The acceleration time $T_{a c c}$ from a standstill up to a frequency set point $f_{s p}$ can be calculated by the following equation:

$$
T_{a c c}=\frac{f_{s p}}{f_{\max }} T_{\text {ramp-up }} .
$$

The synchronous speed is $1500 \mathrm{rpm}$ at the nominal frequency $50 \mathrm{~Hz}$. The maximum frequency is set to $100 \mathrm{~Hz}$. Some examinations were performed at different acceleration time and the same load. Subprogram TRACE and $\mathrm{VC}$ on the drive are used again and the obtained dynamic characteristics are drawn in Fig. 7.
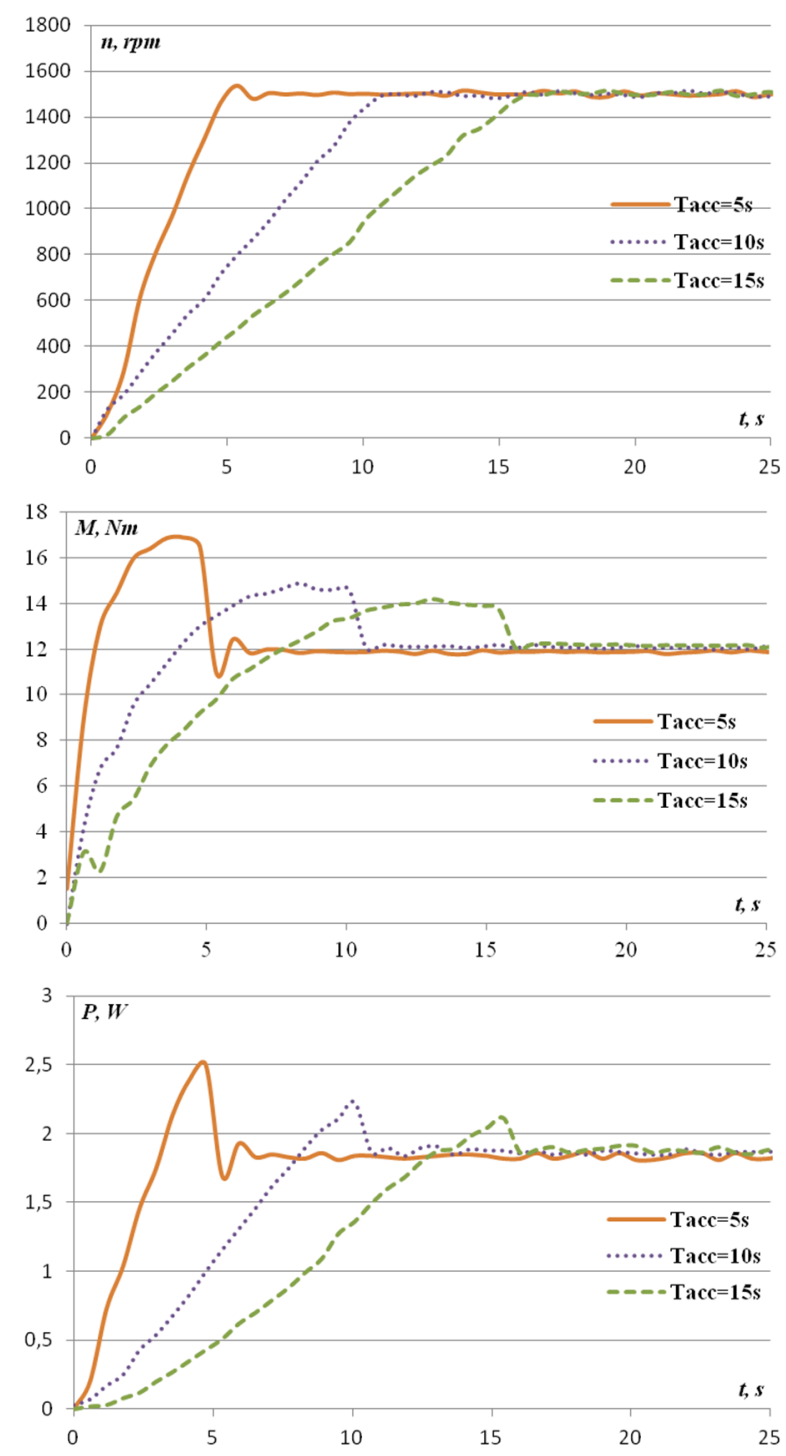

Fig. 7. Dynamic characteristics when starting at different acceleration time.

The main dynamic indicator is the overshoot $\sigma-$ this is the maximum excess of any parameter $y$ over the setpoint value, expressed in percentage: 


$$
\sigma=\frac{y_{M 1}-y_{s p}}{y_{s p}} .100, \%
$$

where: $y_{M 1}$ is the maximum value (exceedance) of the parameter $y$ reached during the transient mode; $y_{s p}$ is the set-point value of this parameter defined by the operator or calculated by the microprocessor CU.

Using (3) the speed, torque, current and power overshoots have been calculated and their dependence on the $T_{a c c}$ is shown in Fig. 8. Analyzing Fig. 7 and Fig. 8 it can be seen that over-speed is negligible (under than 3\%) but could be totally reduced at longer $T_{a c c}$. Shorter acceleration time requires bigger current and torque surges and leads to increased jerk impact on the mechanical part and the power supply (accumulator batteries).

The power consumption also depends on the acceleration time (Fig. 9). Longer $T_{a c c}$ results in lower consumption.

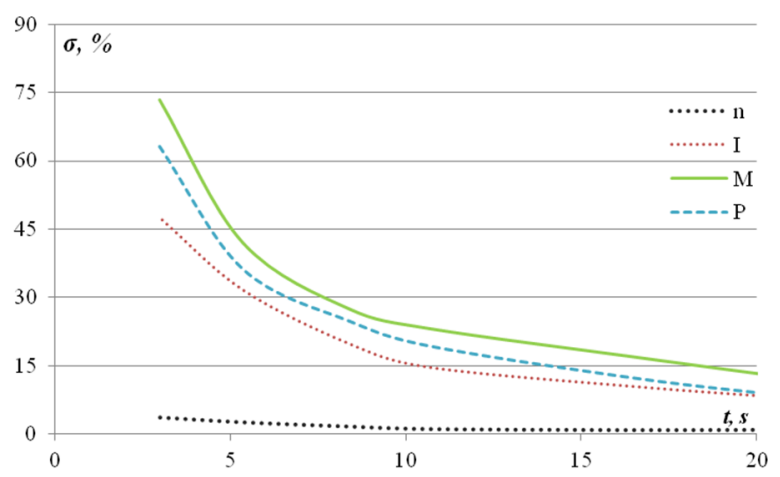

Fig. 8. Graphs representing overshoots dependence on acceleration time.

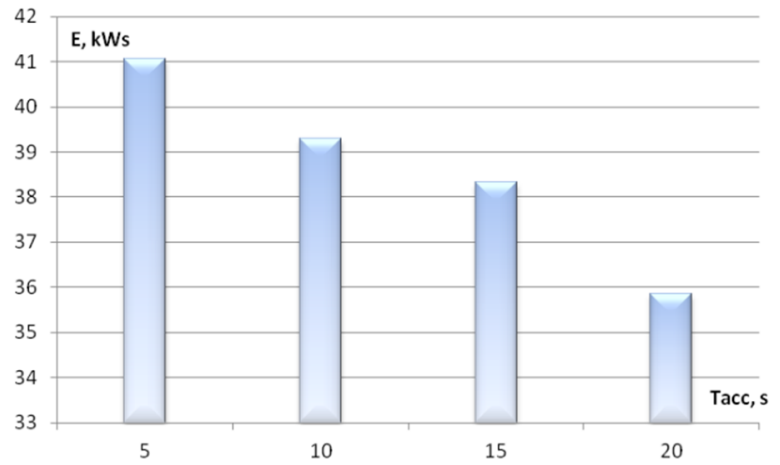

Fig. 9. Graphs representing energy consumption dependence on acceleration time.

In order to avoid torque surges at the transitions from constant velocity phase to accelerating phase, additional rounding-off times could be programmed (parameters "Ramp-up initial rounding time" and "Ramp-up final rounding time"). This is important for electric vehicles, which especially require "soft" (jerk-free) acceleration. In this case, the speed and torque are changed more slightly and smoothly. This leads to increased comfort of the passengers during vehicle acceleration.

Similar tests can be performed to determine the parameters dependence on the deceleration time $T_{d c c}$. A part of the energy can be recuperated (see Fig. 6) and used to recharge the batteries. Shorter $T_{d c c}$ requires bigger overvoltage and breaking torque surges and leads to increased jerk impact and big recuperate current that could damage the batteries or shorten the service life.

The impact of many parameters (PWM frequency, current, torque and speed limitations and many others) on the drive performance could be defined using the developed methodology and the optimal setting of the control system can generally be achieved.

\section{Conclusion}

The implementation of asynchronous motors as a traction machine in electric vehicles provides good performance. Using VC, an infinitely variable speed and torque in a wide range can be realized. The torque and speed fluctuations could be reduced. Very good energetic performance and ride comfort can be achieved.

The developed methodology provides the opportunity of an optimal setting of the drive and combines the advantages of the UDC and SAE J227a driving schedules. The coasting phase is ignored - during it the tractive effort is set to zero but recuperation is not possible, the speed and the energy consumption are not specified, that leads to somewhat of a nuisance to model. The methodology is based on the possibility of examining the influence of the inverter parameters on the energetic drive performance. The optimal ratio of the dynamic features of the car to the energy consumption can be accomplished. The verification has been done using a laboratory simulator the impact of the acceleration time on the drive performance and energy consumption has been defined. This methodology and the obtained results can be used in the design of drives for electric vehicles and their optimal setting - a longest run and maximum passengers comfort can be achieved.

\section{References}

1. L. Balgaranov, Electric transport, Sofia, (2009)

2. U. Bakshi, M. Bakshi, Electrical Drives and Control, (Technical Publications Pune, India, 2009)

3. J. Larminie, J. Lowry, Electric Vehicle Technology Explained, (John Wiley\&Sons Ltd., England 2003)

4. P. Mock, Discrepancies between type-approval and "real-world" fuel-consumption and $\mathrm{CO}_{2}$ values, ICCT Working paper 2012-02 (2012)

5. E/ECE/TRANS/505/Rev.2/Add.100/Rev.3 (12.04.2013)

6. Vehicle Regulations, UNECE Transport Division, http://www.unece.org/trans/main/welcwp29.html

7. V. Dimitrov, E. Dimitrova, Using PLC for control on asynchronous drives - laboratory simulator, ICEST 2016, Ohrid, Macedonia, 471-474 (2016) 